\title{
Comparison of outcome between blood culture positive and negative infective endocarditis patients undergoing cardiac surgery
}

Kristians Meidrops ${ }^{1,2^{*}}$ (D), Arina Zuravlova' ${ }^{1}$, Janis Davis Osipovs ${ }^{1}$, Martins Kalejs ${ }^{1,2}$, Valerija Groma ${ }^{1}$, Eva Petrosina ${ }^{3,4}$, Aigars Reinis ${ }^{5}$, Eva Strike ${ }^{1,6}$, Uga Dumpis $^{7}$, Andrejs Erglis ${ }^{8,9}$ and Peteris Stradins ${ }^{1,2}$

\begin{abstract}
Background: Up to $30 \%$ or even more of all infective endocarditis (IE) cases are recognized as blood culture negative, meaning that the causative agent is left unidentified. The prompt diagnosis together with the identification of causative microorganism and targeted antibiotic treatment can significantly impact the prognosis of the disease and further patient's health status. In some studies, blood culture negative endocarditis has been shown to be associated with delayed diagnosis, worse outcome and course of the disease, and a greater number of intra and postoperative complications.
\end{abstract}

Methods: We retrospectively analysed the medical records of all patients who underwent cardiac surgery for endocarditis between years 2016 and 2019. The aim of this study was to analyse short and long-term mortality and differences of laboratory, clinical and echocardiography parameters in patients with blood culture positive endocarditis (BCPE) and blood culture negative endocarditis (BCNE) and its possible impact on the clinical outcome.

Results: In our study population were 114 (55.1\%) blood culture positive and 93 (44.9\%) blood culture negative cases of infectious endocarditis. The most common pathogens in the blood culture positive IE group were S.aureus in 36 cases (31.6\%), Streptococcus spp. in 27 (23.7\%), E.faecalis in 24 (21.1\%), and other microorganisms in 27 (23.7\%). Embolic events were seen in 60 patients (28.9\%). In univariate analyses, detection of microorganism, elevated levels of procalcitonin were found to be significantly associated with intrahospital death, however it did not reach statistical significance in multivariate analyses. Among microorganisms, S.aureus was significantly associated with intrahospital death in both univariate and multivariate analyses.

Conclusions: There are no statistically significant differences between groups of BCPE and BCNE in terms of intrahospital mortality, hospital and ICU stay or 3-year mortality.

There were higher levels of procalcitonin in BCPE group, however procalcitonin failed to show independent association with mortality in multivariate analysis.

The most common microorganism in the BCPE group was S.aureus. It was associated with independently higher

\footnotetext{
* Correspondence: k.meidrops@gmail.com

'Riga Stradins University, 16 Dzirciema Street, Riga LV-1007, Latvia

${ }^{2}$ Centre of Cardiac Surgery, Pauls Stradins Clinical University Hospital, 13

Pilsonu Street, Riga LV-1002, Latvia

Full list of author information is available at the end of the article
}

(c) The Author(s). 2021 Open Access This article is licensed under a Creative Commons Attribution 4.0 International License, which permits use, sharing, adaptation, distribution and reproduction in any medium or format, as long as you give appropriate credit to the original author(s) and the source, provide a link to the Creative Commons licence, and indicate if changes were made. The images or other third party material in this article are included in the article's Creative Commons licence, unless indicated otherwise in a credit line to the material. If material is not included in the article's Creative Commons licence and your intended use is not permitted by statutory regulation or exceeds the permitted use, you will need to obtain permission directly from the copyright holder. To view a copy of this licence, visit http://creativecommons.org/licenses/by/4.0/ The Creative Commons Public Domain Dedication waiver (http://creativecommons.org/publicdomain/zero/1.0/) applies to the data made available in this article, unless otherwise stated in a credit line to the data. 
intrahospital mortality when compared to other causative microorganisms.

Keywords: Infective endocarditis, Blood culture negative, Blood culture positive, Procalcitonin, Intrahospital mortality, Long-term mortality

\section{Background}

Infective endocarditis (IE) is an infection of the heart valves and inner layer of heart chambers. Even though IE can be caused by any type of bacteria or fungi, the majority of cases are caused by a small number of bacterial species. The most common bacterial species are $S$. aureus, followed by viridans group streptococci and enterococci [1]. Pathogenic bacteria and fungi attach to previously formed lesions and together with platelets and fibrin form vegetations, cause valvular dysfunction, which can manifest as heart failure. Subsequently under the impact of turbulent blood flow, pressure and vegetation instability a rupture may occur in vegetations thus forming emboli. Those in turn may cause septic emboli and ischemic areas in various organ systems $[2,3]$.

In developed countries, the incidence of IE ranges from 4 to 7 cases per 100,000 population per year and has remained stable during recent decades. A relatively rare disease, it still leaves a high impact on an affected patient's life with high mortality rate up to $9-30 \%[2,4]$.

Modified Duke criteria nowadays are being used to diagnose IE. Microbiological detection of a causative agent (MDCA) and positive imaging results are the main criteria. Establishing a diagnosis of definite IE is challenging for physicians as MDCA is not always possible to fulfil.

Up to $30 \%$ or more of IE cases might be blood culture negative. It can be due to low bacteraemia at the moment of blood draw or due to previously initiated antibacterial treatment, fastidious, intracellular pathogens or because the type of medium is not appropriate for some rarer forms of bacteria. It is reported that IE could be even polymicrobial in up to $5 \%$ of cases $[2,5-7]$.

Some studies propose microorganism detection algorithms, which seek to increase microorganism identification level thereby decreasing $\mathrm{BCNE}$ rate. It should be considered whether microorganism detection by these specific algorithms is clinically relevant and cost effective in cases where diagnosis of IE can be made based on ordinary imaging and clinical findings. If any additional detection methods are being used, it is important to determine whether possible results will significantly change a treatment plan. Patient risk factor identification and atypical causative microorganism detection could be beneficial for developing a more precise and effective treatment strategy depending on the clinical situation [8].

The prompt diagnosis together with identification of IE causative agent and targeted antimicrobial treatment can significantly impact the prognosis of the disease and further on patient's health status. In some studies, blood culture negative endocarditis is associated with delayed diagnosis, worse outcome and course of the disease, greater number of intra- and postoperative complications $[8,9]$.

On the other hand, there are studies which suggest that $\mathrm{BCNE}$ and $\mathrm{BCPE}$ have no difference in short and long-term survival. These papers note that other independent factors such as diabetes mellitus contribute more to the clinical course than microorganism detection alone $[10,11]$.

Other authors also point out certain laboratory findings that could be more commonly seen in BCNE such as lower CRP levels and higher BNP levels $[12,13]$.

Therefore, the aim of this study was to analyse short and long-term mortality and differences of laboratory, clinical and echocardiography parameters in patients with $\mathrm{BCPE}$ and $\mathrm{BCNE}$ and its possible impact on the clinical outcome.

\section{Methods}

We conducted a retrospective analysis of medical records of all 207 IE patients who underwent cardiac surgery at Pauls Stradins Clinical University Hospital, Latvia, between years 2016 and 2019. Our centre is the only one providing adult cardiac surgery in Latvia thus representing the whole surgically treated infective endocarditis patient population in Latvia. Two groups of patients were defined - patients with BCPE and patients with BCNE. In our hospital no Endocarditis team was available during the time of the study. Diagnosis of IE was made by cardiologists and cardiac surgeons and specialists from different specialities depending on patients' clinical status and comorbidities. Definite and possible IE diagnosis was based on modified Duke criteria.

All the data from laboratory analyses refer to the last samples taken prior surgery. The study was approved by the Ethical Committee of the Hospital (Decision No. 230419-17 L).

\section{Statistical analysis}

Categorical variables were expressed as relative frequencies (percentages), for quantitative variables mean (standard deviation [SD]) and median (interquartile range $[\mathrm{IQR}]$ ) were used for description of normally and non-normally distributed data, respectively. Graphical tools were used for assessing normality. For categorical variables accordingly to tests assumptions Chi-square 
Table 1 Indications for cardiac surgery in patients with IE

\begin{tabular}{ll}
\hline Heart failure due to valvular dysfunction & $5.80 \%$ \\
Prevention of embolism & $3.86 \%$ \\
$\begin{array}{l}\text { Heart failure due to valvular dysfunction } \\
\text { and prevention of embolism }\end{array}$ & $41.06 \%$ \\
$\begin{array}{l}\text { Heart failure due to valvular dysfunction } \\
\text { and prevention of embolism and uncontrolled } \\
\text { infection }\end{array}$ & $28.02 \%$ \\
$\begin{array}{l}\text { Heart failure due to valvular dysfunction and } \\
\text { uncontrolled infection }\end{array}$ & $20.29 \%$ \\
Uncontrolled infection and prevention of embolism & $0.97 \%$
\end{tabular}

test or Fisher exact test was used to compare difference between groups. For quantitative variables accordingly to test assumptions t-test or Mann-Whitney $U$ test was used to compare groups. Univariate and multivariate logistic regression was used to see association between potential factors that might affect survival (intrahospital death). $P$-values below 0.05 were considered statistically significant. Statistical analyses were performed using IBM SPSS version 26. RStudio, version 1.3.1073 was used to depict the results of survival analysis.

\section{Results}

We collected medical records of 207 patients who underwent cardiac surgery for IE. There were 114 (55.1\%) blood culture positive and 93 (44.9\%) blood culture negative cases of infectious endocarditis. The rate of pathogen detection from tissue cultures obtained at the time of surgery was low. In the BCNE group pathogen from tissue culture was detected in 5.4 and $13.2 \%$ in the BCPE group.

Table 2 Characteristics of IE patients depending on blood culture status

\begin{tabular}{|c|c|c|c|}
\hline & $\begin{array}{l}\text { Blood culture } \\
\text { positive }\end{array}$ & $\begin{array}{l}\text { Blood culture } \\
\text { negative }\end{array}$ & $\begin{array}{l}p \\
\text { value } \\
\end{array}$ \\
\hline Age, mean (years) & $57.17(15.59)$ & $53.61(12.80)$ & 0.073 \\
\hline Sex, male, \% & 78.07 & 65.59 & 0.046 \\
\hline BMI, mean $\left(\mathrm{kg} / \mathrm{m}^{2}\right)$ & $25.45(4.48)$ & $25.91(5.65)$ & 0.531 \\
\hline PVE, \% & 21.51 & 19.30 & 0.694 \\
\hline Aortic valve IE, \% & 38.60 & 41.94 & 0.626 \\
\hline Mitral valve IE, \% & 32.46 & 22.58 & 0.116 \\
\hline $\begin{array}{l}\text { Aortic and mitral valve } \mathrm{IE}_{\mathrm{E}} \\
\%\end{array}$ & 18.42 & 29.03 & 0.072 \\
\hline Left heart side IE, \% & 89.47 & 95.70 & 0.095 \\
\hline $\begin{array}{l}\text { Intravenous drug injection } \\
\text { history, \% }\end{array}$ & 12.28 & 4.30 & 0.043 \\
\hline $\begin{array}{l}\text { Haemodynamically stable, } \\
\%\end{array}$ & 88.60 & 92.47 & 0.348 \\
\hline $\begin{array}{l}\text { Left ventricle ejection } \\
\text { fraction, mean, } \%\end{array}$ & $55.24(11.19)$ & $55.77(9.18)$ & 0.714 \\
\hline Euroscore II risk, mean, \% & 7.35 & 6.80 & 0.595 \\
\hline
\end{tabular}

Table 3 Comorbidities in BCPE and BCNE patients

\begin{tabular}{llll}
\hline & $\begin{array}{l}\text { Blood culture } \\
\text { positive }\end{array}$ & $\begin{array}{l}\text { Blood culture } \\
\text { negative }\end{array}$ & $\begin{array}{l}\boldsymbol{p} \\
\text { value }\end{array}$ \\
\hline $\begin{array}{l}\text { Diabetes mellitus, } \\
\text { type 1, \% }\end{array}$ & 5.56 & 0.00 & $\mathbf{0 . 0 3 2}$ \\
$\begin{array}{l}\text { Diabetes mellitus, } \\
\text { type 2, \% }\end{array}$ & 9.26 & 8.79 & 0.909 \\
HIV infection, \% & 6.42 & 1.10 & 0.074 \\
HCV infection, \% & 15.74 & 5.49 & $\mathbf{0 . 0 2 2}$ \\
HBV infection, \% & 0.00 & 1.10 & 0.457 \\
Spondylodiscitis, \% & 5.26 & 1.08 & 0.132 \\
Oncology, \% & 4.39 & 3.23 & 0.733 \\
Arterial hypertension, & 4.59 & 12.09 & 0.720 \\
\% & & & \\
\hline
\end{tabular}

Preoperatively all BCPE cases and 71 of 93 (76.3\%) BCNE cases were classified as definite infective endocarditis. Twenty-two cases of BCNE were classified as possible infective endocarditis and met definitive endocarditis criteria when confirmed histologically.

Necessity for cardiac surgery was based on three main indications suggested in 2015 ESC European guidelines for the management of infective endocarditis - heart failure, uncontrolled infection and prevention of embolism. As shown in Table 1, indications for cardiac surgery most often were combined, leaving isolated indication such as heart failure due to valvular dysfunction and isolated prevention of embolism only in the minority of cases of left heart side IE.

Table 2 summarises the patients' characteristics. In the BCPE group there were significantly higher number of patients with intravenous drug addiction as well as a prevalence of $\mathrm{HCV}$ infection. Among comorbidities type 1 diabetes mellitus rate was significantly higher in BCPE group patients (Table 3 ). The most commonly detected pathogens were S.aureus in 36 (31\%) cases, Streptococcus spp. in 27 (24\%), E.faecalis in 24 (21\%) and other microorganisms in 27 (24\%) cases. There was no statistically significant difference between groups of

Table 4 Occurrence of embolic events, locally uncontrolled infection, haemodynamic instability prior surgery and vegetation size in IE patients depending on blood culture status

\begin{tabular}{llll}
\hline & $\begin{array}{l}\text { Blood culture } \\
\text { positive }\end{array}$ & $\begin{array}{l}\text { Blood culture } \\
\text { negative }\end{array}$ & $\begin{array}{l}\boldsymbol{p} \\
\text { value }\end{array}$ \\
\hline $\begin{array}{l}\text { Embolic events prior to } \\
\text { surgery, \% }\end{array}$ & 29.82 & 27.96 & 0.768 \\
$\begin{array}{l}\text { Locally uncontrolled } \\
\text { infection, \% }\end{array}$ & 30.00 & 31.87 & 0.775 \\
$\begin{array}{l}\text { Haemodynamically } \\
\text { unstable, \% }\end{array}$ & 11.40 & 7.53 & 0.348 \\
$\begin{array}{l}\text { Vegetation size, mm, } \\
\text { mean }\end{array}$ & $16.70(9.95)$ & $14.48(7.69)$ & 0.097 \\
\hline
\end{tabular}


Table $\mathbf{5}$ Laboratory analyses in IE patients depending on blood culture status

\begin{tabular}{llll}
\hline & Blood culture positive & Blood culture negative & $\boldsymbol{p}$ value \\
\hline CRP, mg/L & $37.90(9.98-94.33)$ & $25.00(12.25-63.70)$ & 0.206 \\
Procalcitonin, $\mathrm{ng} / \mathrm{ml}$ & $0.45(0.10-2.33)$ & $0.10(0.10-0.50)$ & $\mathbf{0 . 0 0 1}$ \\
BNP, pg/ml & $560.45(157.73-1580.05)$ & $469.50(178.35-1114.30)$ & 0.424 \\
Creatinine, $\mathrm{mmol} / \mathrm{L}$ & $85.50(63.25-107.75)$ & $87.50(66.00-110.00)$ & 0.734 \\
Glucose, $\mathrm{mmol} / \mathrm{L}$ & $6.08(1.70)$ & $6.20(1.56)$ & 0.614 \\
Bilirubin, $\mu \mathrm{mol} / \mathrm{L}$ & $8.00(6.00-15.00)$ & $8.00(6.00-12.00)$ & 0.592 \\
Leukocyte count $\times 10 \wedge 9 / \mathrm{L}$ & $9.20(4.15)$ & $8.81(3.73)$ & $\mathbf{0 . 4 8 4}$ \\
Haemoglobin, $\mathrm{g} / \mathrm{L}$ & $103.85(23.00)$ & $110.91(21.09)$ & $\mathbf{0 . 0 2 4}$ \\
Haematocrit, $\%$ & $32.12(6.07)$ & $34.06(6.41)$ & $\mathbf{0 . 0 2 9}$ \\
Platelet count $\times 10 \wedge 9 / \mathrm{L}$ & $261.73(129.83)$ & $256.58(110.58)$ & 0.763
\end{tabular}

Mean (SD) or Med (IQR) used

$\mathrm{BCPE}$ and $\mathrm{BCNE}$ regarding locally uncontrolled infection, vegetation size, embolic events and haemodynamic instability (Table 4). Even though patients with embolic events had bigger vegetation size, we did not find significant association. Mean vegetation size among patients who had embolic events was 16.0 (11.3) $\mathrm{mm}$ versus $15.5(7.9) \mathrm{mm}$ among those who did not $(p=$ 0.795). Altogether embolic events were seen in 60 patients (28.9\%). In laboratory analyses, significantly higher levels of procalcitonin and lower levels of haemoglobin and haematocrit were observed in the BCPE group (Table 5). Intrahospital mortality in the BCPE group was $14.04 \%$ in comparison with $5.38 \%$ in the BCNE group, however it did not reach statistical significance $(p=0.062)$ (Table 6).

Microorganism detection and higher procalcitonin level in univariate analysis showed an association with intrahospital death, however it did not feature as an independent predictor of mortality in multivariate analysis (Table 7). Among microorganisms, S.aureus was independently associated with intrahospital mortality (Table 8). In long-term period (Fig. 1) better survival was seen in BCNE, however, statistical significance was not found $(p=0.509)$.

\section{Discussion}

In this retrospective study, we analysed $\mathrm{BCPE}$ and $\mathrm{BCNE}$ patients' data and clinical outcomes undergoing cardiac surgery. Of 207 patients, 93 (44.9\%) were BCNE. In literature, the rate of $\mathrm{BCNE}$ is described as greatly varying from 2.5 up to $70 \%$, indicating a high rate of $\mathrm{BCNE}$ in this study [14].

In the study of Fournier et al. the rate of BCNE within a prospective analysis of 918 patients was $30.8 \%$. Their proposed diagnostic strategy in 283 patients with $\mathrm{BCNE}$ found aetiology in 138 patients (78.0\%) in whom 135 had causative microorganism identified. Of these, intracellular bacteria as C.burnetii, Bartonella spp, and T.whipplei accounted for $15.9 \%$ cases of BCNE. In 3 patients (1.1\%), the aforementioned authors confirmed non-infectious aetiology of endocarditis. In BCNE, 56 of 70 patients infected with gram-positive cocci had received antibiotics prior to blood culture collection, indicating early antibiotics administration as a possible cause of BCNE [8]. During the time period of the study we used conventional methods of bacteriological examination of culturable pathogens without any extended diagnostic strategy, which partly explains the high incidence of BCNE and low detection of intracellular bacteria. Data published by Lamas et al. suggested that $\mathrm{BCNE}$ resulted mostly from priorly administered antibiotics and had an association with severe haemodynamic compromise [11]. Since many patients were transferred to our hospital from different institutions, we failed to assess priorly administered antibiotics from medical records. This could play a major role in our study, resulting in lower inflammatory markers and less severe preoperative condition in BCNE group.

Table 6 Duration of hospital and ICU stay, reoperation for bleeding and mortality rates in IE patients depending on blood culture status

\begin{tabular}{llll}
\hline & Blood culture positive & Blood culture negative & $\boldsymbol{p}$ value \\
\hline Intrahospital stay, days, mean & $25.00(16.00-35.00)$ & $23.00(17.50-31.00)$ & 0.378 \\
Stay in ICU, days, mean & $2.00(1.00-5.00)$ & $2.00(1.00-3.00)$ & 0.828 \\
Reoperation for bleeding, \% & 17.54 & 12.90 & 0.358 \\
Mortality, \% & 14.04 & 5.38 & 0.062 \\
\hline
\end{tabular}


Table 7 Predictors of hospital mortality by univariable and multivariable logistic regression analyses

\begin{tabular}{lllll}
\hline Variable & Univariable OR $(\mathbf{9 5} \% \mathrm{Cl})$ & $\boldsymbol{p}$ value & Multivariable adjusted OR (95\% Cl) & $\boldsymbol{p}$ value \\
\hline Microorganism detection & $2.873(1.011-8.167)$ & $\mathbf{0 . 0 4 8}$ & $1.166(0.319-4.272)$ & 0.816 \\
Age & $1.024(0.991-8.167)$ & 0.155 & $1.027(0.975-1.082)$ & 0.319 \\
Body mass index & $1.034(0.946-1.129)$ & 0.466 & $1.034(0.912-1.173)$ & 0.599 \\
CRP (C-reactive protein) & $1.003(0.995-1.011)$ & 0.490 & $1.002(0.991-1.014)$ & 0.696 \\
Procalcitonin & $1.036(1.004-1.068)$ & $\mathbf{0 . 0 2 5}$ & $1.033(0.999-1.068)$ & 0.057 \\
Leukocytes & $1.029(0.924-1.146)$ & 0.608 & $1.066(0.909-1.249)$ & 0.433 \\
Embolism & $1.586(0.621-4.048)$ & 0.335 & $1.311(0.368-4.673)$ & 0.677 \\
Intravenous drug user & $0.497(0.063-3.937)$ & 0.508 & $1.202(0.087-16.600)$ & 0.891 \\
Prosthetic valve endocarditis & $2.157(0.810-5.742)$ & 0.124 & $0.849(0.024-29.994)$ & 0.928 \\
Native valve endocarditis & $0.464(0.174-1.234)$ & 0.124 & $0.840(0.025-27.790)$ & 0.922
\end{tabular}

Data in literature for BCNE patients regarding the outcome are controversial. Our study findings indicate no statistically significant difference of intrahospital mortality and long-term mortality between BCPE and BCNE patients. However, there was a tendency towards a worse outcome in the $\mathrm{BCPE}$ group. In univariate analysis there were higher rates of intrahospital mortality, although it failed to show statistical significance in multivariate analysis. This finding can possibly be explained by a higher severity of preoperative condition and less controlled infection in the BCPE group. We observed significantly lower haemoglobin and haematocrit levels prior to surgery, a higher procalcitonin level (Table 5) and other factors such as drug addiction (Table 2) and the presence of comorbidities (Table 3). Whilst not statistically significant, there were higher rates of haemodynamic instability and higher CRP levels in the current study. Our findings are in line with the results observed by Phua et al. characterizing outcomes of culture negative versus culture positive sepsis [15].

Higher inflammatory markers such as CRP and procalcitonin are usually associated with more severe preoperative conditions. The usefulness of a procalcitonin marker has been explored by other authors when studying IE and sepsis [16-18]. Recently, procalcitonin has been recognized as the most studied and suitable biomarker regarding antibiotic stewardship $[19,20]$. There are studies suggesting that higher baseline CRP levels are independently associated with short term adverse events and higher procalcitonin levels are independently associated with increased intrahospital mortality [12, 21]. R.F.Siciliano et al., in a prospective study of 221 episodes, BCNE and BCPE were compared and a $\mathrm{BCNE}$ rate of $23.1 \%$ was reported. They have found lower CRP levels at the time of admission in BCNE group, which is consistent with the results of our study [13]. Our study showed an increased procalcitonin level in association with mortality in the univariate analysis, however it failed to show it in the multivariate analysis (Table 7).

The most commonly detected microorganism was S.aureus (31.6\%), which was significantly associated with intrahospital death among other microorganisms in both univariate and multivariate analyses (Table 8). This finding is in agreement with the results demonstrated by other authors [22]. S.aureus is a pathogen known for its aggressive clinical presentation and worse prognosis compared to other microorganisms [23]. A high proportion of aggressive microorganism such as S.aureus in BCPE group could impact outcome results and might be associated with more severe preoperative conditions. Multi-antibiotic resistance in our study did not seem to impact the outcome, since there were only few multiresistant microorganism caused IE cases.

Two major limitations of this study are the relatively small sample size and a single center study. There are however very few studies published regarding this topic, especially studying the role of BCNE among the patients' undergoing cardiac surgery. Our cardiac surgery center is the only one providing adult cardiac surgery services in the country and is therefore representing the whole

Table 8 Detected microorganisms as predictors of hospital mortality by univariable and multivariable logistic regression analyses

\begin{tabular}{lllll}
\hline Variable & Univariable OR $\mathbf{( 9 5 \% ~ C l )}$ & $\boldsymbol{p}$ value & Multivariable adjusted OR $(\mathbf{9 5 \%} \mathbf{C l})$ & $\boldsymbol{p}$ value \\
\hline E.faecalis & $2.325(0.527-10.246)$ & 0.265 & $1.310(0.356-4.824)$ & 0.685 \\
Streptococcus spp. & $1.788(0.422-7.587)$ & 0.430 & $1.073(0.295-3.910)$ & 0.915 \\
S.aureus & $4.408(1.406-13.821)$ & $\mathbf{0 . 0 1 1}$ & $3.332(1.268-8.751)$ & $\mathbf{0 . 0 1 5}$ \\
Other microorganism & $1.302(0.243-6.962)$ & 0.758 & $0.678(0.149-3.089)$ & 0.615 \\
\hline
\end{tabular}




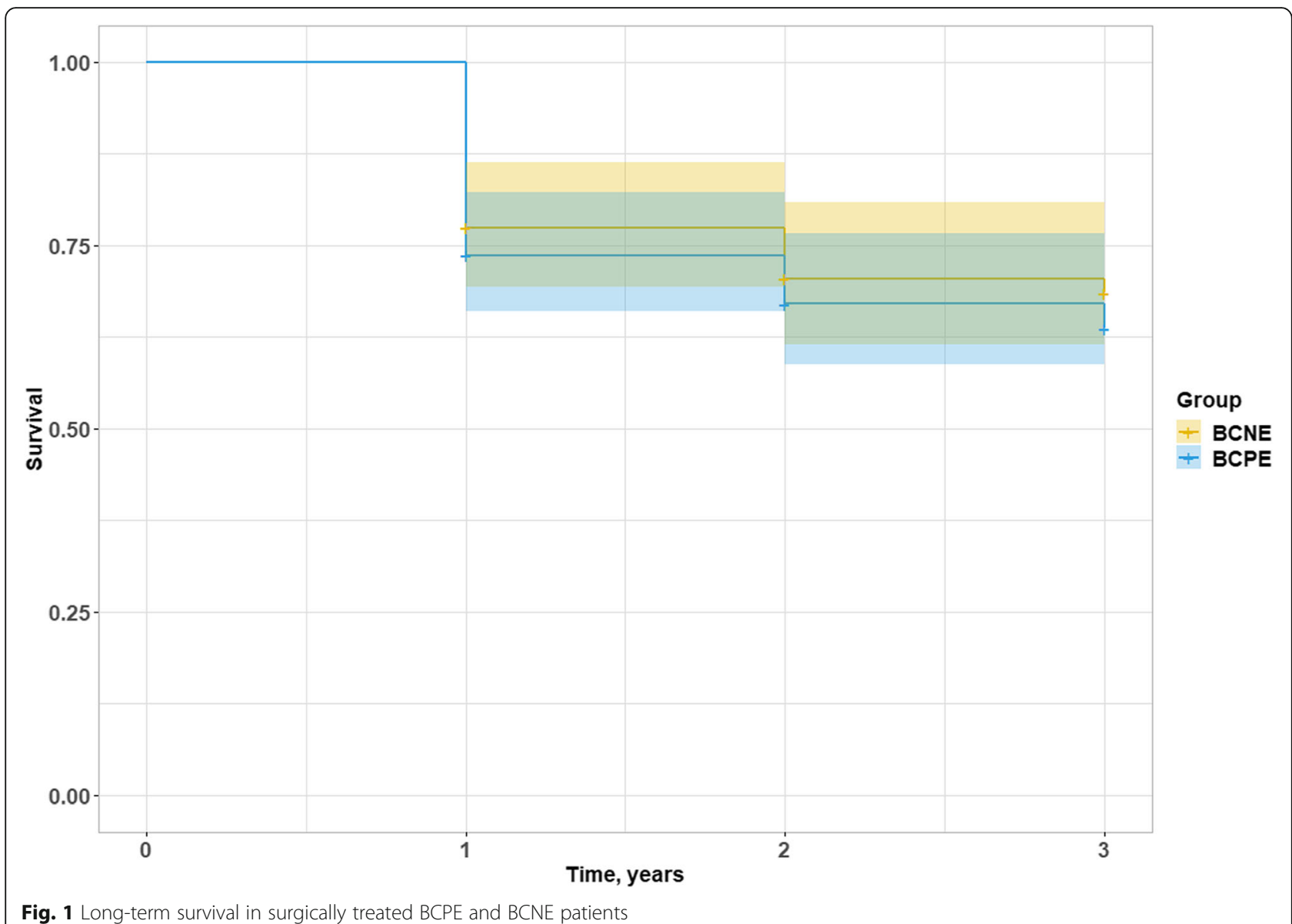

surgically treated infective endocarditis patient population in Latvia.

\section{Conclusions}

There are no statistically significant differences between groups of BCPE and BCNE in terms of intrahospital mortality, hospital and ICU stay or 3-year mortality.

Although BCPE patients have higher intrahospital and long-term mortality than BCNE patients, BCPE is not independently associated with mortality in multivariate analysis.

There were higher levels of procalcitonin in BCPE group, however procalcitonin failed to show independent association with mortality in multivariate analysis.

The most common microorganism in the BCPE group was S.aureus. It was associated with independently higher intrahospital mortality (OR rate of 3.332 and 4.408 in uni- and multivariate analyses) when compared to other causative microorganisms.

\section{Abbreviations}

IE: Infective endocarditis; BCPE: Blood culture positive infective endocarditis; BCNE: Blood culture negative infective endocarditis; NVE: Native valve endocarditis; PVE: Prosthetic valve endocarditis; CRP: C-reactive protein: MDCA: Microbial detection of causative agent; SD: Standard deviation;
IQR: Interquartile range; HIV: Human immunodeficiency virus; HCV: Hepatitis C virus; HBV: Hepatitis B virus; BMI: Body mass index; ICU: Intensive care unit; BNP: B-type natriuretic peptide

\section{Acknowledgements}

Not applicable.

\section{Authors' contributions}

$\mathrm{KM}, \mathrm{AZ}$, JDO collected the data and prepared the manuscript, KM did the formal analysis, EP did the statistical analysis, KM, AZ, JDO prepared the original draft, VG, MK, PS supervised the study, did the writing, review, and editing of the draft, $A R, E S, U D, A E$ did the editing and interpretation of data. All authors have read and agreed to the published version of the manuscript. The author(s) read and approved the final manuscript.

\section{Funding}

This study didn't receive any funding.

Availability of data and materials

The dataset used and analysed during the current study is available from the corresponding author upon reasonable request.

\section{Declarations}

Ethics approval and consent to participate

This study was approved by the Ethical Committee of Pauls Stradins Clinical University Hospital. Protocol number 230419-17 L.

Consent for publication

Not applicable. 


\section{Competing interests}

The authors declare that they have no competing interests.

\section{Author details}

${ }^{1}$ Riga Stradins University, 16 Dzirciema Street, Riga LV-1007, Latvia. ${ }^{2}$ Centre of Cardiac Surgery, Pauls Stradins Clinical University Hospital, 13 Pilsonu Street, Riga LV-1002, Latvia. ${ }^{3}$ Statistics Unit, Riga Stradins University, 14 Balozu Street, Riga LV-1007, Latvia. ${ }^{4}$ Faculty of Physics, Mathematics and Optometry, UL House of Science, University of Latvia, 3 Jelgavas Street, Riga LV-1004, Latvia. ${ }^{5}$ Department of Biology and Microbiology, Riga Stradins University, 16 Dzirciema Street, Riga LV-1007, Latvia. ${ }^{6}$ Department of Cardiovascular Anaesthesia and Intensive Care, Pauls Stradins Clinical University Hospital, Riga LV-1002, Latvia. 'Department of Infection Control, Pauls Stradins Clinical University Hospital, 13 Pilsonu Street, Riga LV-1002, Latvia. ${ }^{8}$ Centre of Cardiology, Pauls Stradins Clinical University Hospital, 13 Pilsonu Street, Riga LV-1002, Latvia. ${ }^{9}$ Faculty of Medicine, UL House of Science, University of Latvia, 3 Jelgavas Street, Riga LV-1004, Latvia.

Received: 14 December 2020 Accepted: 7 May 2021

Published online: 27 May 2021

\section{References}

1. Jong EC, Stevens DL, Netter FH. Netter's infectious diseases. Philadelphia: Elsevier/Saunders; 2012.

2. Habib G. Management of infective endocarditis. Heart. 2006;92(1):124-30. https://doi.org/10.1136/hrt.2005.063719.

3. Habib G, Lancellotti P, Antunes MJ, Bongiorni MG, Casalta J-P, Del Zotti F, et al. 2015 ESC guidelines for the management of infective endocarditis: the task force for the Management of Infective Endocarditis of the European Society of Cardiology (ESC) endorsed by: European Association for CardioThoracic Surgery (EACTS), the European as. Eur Heart J. 2015;36(44):3075128. https://doi.org/10.1093/eurheartj/ehv319.

4. Hsieh F-C, Huang C-Y, Lin S-F, Sun J-T, Yen T-H, Chang C-C. Short-term exposure to particulate matters is associated with septic emboli in infective endocarditis. Medicine (Baltimore). 2019;98(45):e17899. https://doi.org/10.1 097/MD.0000000000017899.

5. Thuny F, Grisoli D, Collart F, Habib G, Raoult D. Management of infective endocarditis: challenges and perspectives. Lancet. 2012;379(9819):965-75.

6. Bosshard PP, Kronenberg A, Zbinden R, Ruef C, Böttger EC, Altwegg M. Etiologic diagnosis of infective endocarditis by broad-range polymerase chain reaction: a 3-year experience. Clin Infect Dis. 2003;37(2):167-72. https://doi.org/10.1086/375592.

7. Miyazato A, Ohkusu K, Tabata M, Uwabe K, Kawamura T, Tachi Y, et al. Comparative molecular and microbiological diagnosis of 19 infective endocarditis cases in which causative microbes were identified by PCRbased DNA sequencing from the excised heart valves. I Infect Chemother. 2012;18(3):318-23. https://doi.org/10.1007/s10156-011-0332-0.

8. Fournier P-E, Gouriet F, Casalta J-P, Lepidi H, Chaudet H, Thuny F, et al. Blood culture-negative endocarditis: improving the diagnostic yield using new diagnostic tools. Medicine (Baltimore). 2017;96(47):e8392. https://doi. org/10.1097/MD.0000000000008392.

9. Alberto SRJ, Isidre V, Javier L, Cristina S. Critical questions about left-sided infective endocarditis. J Am Coll Cardiol. 2015;66(9):1068-76.

10. Ferrera C, Vilacosta I, Fernández C, López J, Olmos C, Sarriá C, et al. Reassessment of Blood Culture-Negative Endocarditis: Its Profile Is Similar to That of Blood Culture-Positive Endocarditis. Rev Española Cardiol (English Ed). 2012;65(10):891-900

11. Lamas CC, Fournier P-E, Zappa M, Brandão TJD, Januário-da-Silva CA Correia MG, et al. Diagnosis of blood culture-negative endocarditis and clinical comparison between blood culture-negative and blood culturepositive cases. Infection. 2016;44(4):459-66. https://doi.org/10.1007/s1501 0-015-0863-x.

12. Mohanan S, Gopalan Nair R, Vellani H, Sajeev CG, George B, Krishan MN. Baseline C-reactive protein levels and prognosis in patients with infective endocarditis: A prospective cohort study. Indian Heart J. 2018;70:543-9.

13. Siciliano RF, Gualandro DM, Bittencourt MS, Paixão M, Marcondes-Braga F, de Soeiro AM, et al. Biomarkers for prediction of mortality in left-sided infective endocarditis. Int J Infect Dis. 2020;96:25-30.

14. Brouqui P, Raoult D. Endocarditis Due to Rare and Fastidious Bacteria. Clin Microbiol Rev. 2001;14(1):177 LP-207.
15. Phua J, Ngerng WJ, See KC, Tay CK, Kiong T, Lim HF, et al. Characteristics and outcomes of culture-negative versus culture-positive severe sepsis. Crit Care. 2013;17(5):R202.

16. Schuetz P, Albrich W, Mueller B. Procalcitonin for diagnosis of infection and guide to antibiotic decisions: past, present and future. BMC Med. 2011;9(1): 107. https://doi.org/10.1186/1741-7015-9-107.

17. Sridharan $\mathrm{P}$, Chamberlain RS. The efficacy of procalcitonin as a biomarker in the management of sepsis: slaying dragons or tilting at windmills? Surg Infect. 2013;14(6):489-511. https://doi.org/10.1089/sur.2012.028.

18. Azzini AM, Dorizzi RM, Sette P, Vecchi M, Coledan I, Righi E, et al. A 2020 review on the role of procalcitonin in different clinical settings: an update conducted with the tools of the evidence based laboratory medicine. Ann Transl Med. 2020;8(9):610. https://doi.org/10.21037/atm-20-1855.

19. Neeser O, Branche A, Mueller B, Schuetz P. How to: implement procalcitonin testing in my practice. Clin Microbiol Infect Off Publ Eur Soc Clin Microbiol Infect Dis. 2019;25(10):1226-30.

20. Gregoriano C, Heilmann E, Molitor A, Schuetz P. Role of procalcitonin use in the management of sepsis. J Thorac Dis. 2020;12(Suppl 1):S5-15. https://doi. org/10.21037/jtd.2019.11.63.

21. AlRawahi AN, AlHinai FA, Doig CJ, Ball CG, Dixon E, Xiao Z, et al. The prognostic value of serum procalcitonin measurements in critically injured patients: a systematic review. Crit Care. 2019;23(1):390. https://doi.org/10.11 86/s13054-019-2669-1

22. Wang A, Gaca JG, Chu VH. Management considerations in infective endocarditis: a review. JAMA. 2018;320(1):72-83. https://doi.org/10.1001/ja ma.2018.7596.

23. Han SM, Sorabella RA, Vasan S, Grbic M, Lambert D, Prasad R, et al. Influence of Staphylococcus aureus on Outcomes after Valvular Surgery for Infective Endocarditis. J Cardiothorac Surg. 2017;12(1):57.

\section{Publisher's Note}

Springer Nature remains neutral with regard to jurisdictional claims in published maps and institutional affiliations.
Ready to submit your research? Choose BMC and benefit from:

- fast, convenient online submission

- thorough peer review by experienced researchers in your field

- rapid publication on acceptance

- support for research data, including large and complex data types

- gold Open Access which fosters wider collaboration and increased citations

- maximum visibility for your research: over 100M website views per year

At $\mathrm{BMC}$, research is always in progress.

Learn more biomedcentral.com/submissions 